\title{
The emulsifier fault early warning on BP neural network theory
}

\author{
Yuesheng Wang ${ }^{1, a}$, Kangjian $\mathrm{Lu}^{2, \mathrm{~b}}$ \\ ${ }^{1}$ Institute of Automation, Hangzhou Dianzi University, Hangzhou Zhejiang 310018, China; \\ ${ }^{2}$ Institute of Automation, Hangzhou Dianzi University, Hangzhou Zhejiang 310018, China. \\ awys@hdu.edu.cn, b442128310@qq.com
}

Keywords: emulsifier, fault warning, BP neural network

\begin{abstract}
Emulsifier is the key equipment in the production of emulsion explosive. The condition of the emulsifier is very important to the production safety of the emulsion explosive. The paper focuses on emulsifier fault warning in the production of emulsion explosive. Now the emulsifier fault warning is based on the threshold. That method can not warn fault timely. The paper proposes a fault early warning method which is on the BP neural network. The neural network model is established by the method. The condition of the emulsifier which is important to the production safety can be predicted through the model. According to the simulation example, the model can predict the conduction of the emulsifier well.
\end{abstract}

\section{Introduction}

Emulsifier is the key equipment in the production of emulsion explosive. Emulsifier fault is the main cause of the most production safety accidents in the past ten years. So it is important to warning emulsifier fault. The existing fault warning is on the monitoring parameters. Fault warning is triggered if the parameters exceed the threshold [1]. While the accident causes by the emulsifier fault has often happened. The method is not effective to warn fault in time.

To mix the oil phase solution and aqueous solution adequately, emulsifier runs in high speed. The temperature and flow rate of the oil phase solution or aqueous solution affect the condition of emulsifier. It is also affected by the pressure and current of the emulsifier [2]. Combining with the working principle of the emulsifier, the paper proposes a fault warning method on the BP neural network.

\section{Fault early warning process}

A large numbers of historical data about production line of emulsion explosive is stored in the configuration software. Based on the BP neural network and the historical data, a model is built. This method for fault warning is the improvement of emulsifier threshold alarm used widely now. Fig.1 shows the process for emulsifier fault early warning.

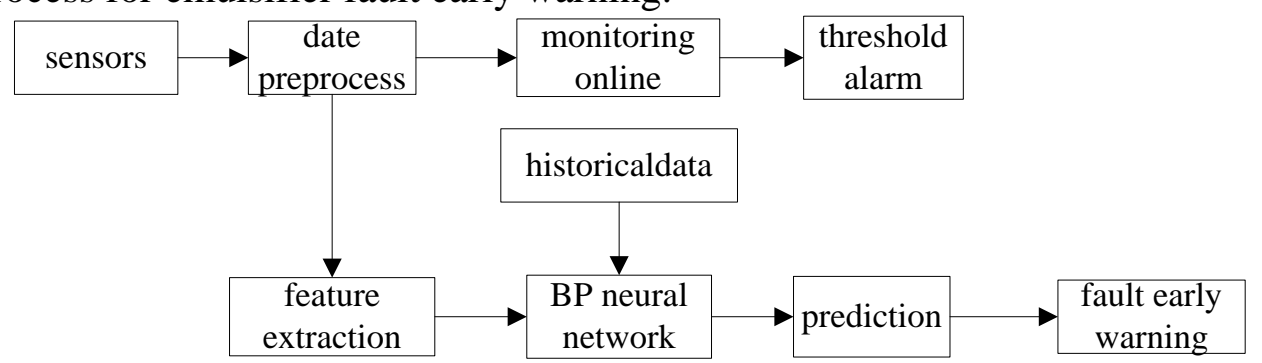

Fig.1 Fault early warning process

\section{The selection of research object}

Emulsifier is a kind of rotating equipment. It produces emulsion matrix by stirring specific solution and oil phase. During the stirring process, emulsifier vibrates. The amplitude of 
vibration is controlled within certain range in normal conditions. Abnormal vibration happens when something goes wrong with the emulsifier. So vibration is selected as the main index to show the condition of the emulsifier. According to the operation of the emulsifier, the other 7 parameters which are effected the vibration are selected for emulsifier fault early warning. The 7 parameters are the temperature and flow rate of the oil phase solution or aqueous solution, the pressure and current of the emulsifier, the temperature of the emulsion matrix.

\section{BP neural network algorithm}

Artificial neurons are the basic unit of the BP neural network. Fig.2 shows an artificial neurons model.

In the Fig.2, $\mathrm{x}_{\mathrm{i}}$ is one of input and $\mathrm{y}_{\mathrm{i}}$ is one of the output, $\mathrm{s}_{\mathrm{i}}$ is the input which is outside the neurons, $\mathrm{w}_{\mathrm{ij}}$ is the weight between two artificial neurons [3].

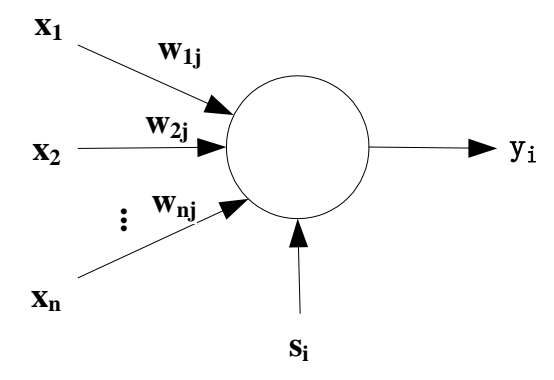

Fig.2 The artificial neurons model

The formula (1) and (2) show the relation of $\mathrm{x}_{\mathrm{i},} \mathrm{y}_{\mathrm{i}}, \mathrm{s}_{\mathrm{i}}$ and $\mathrm{w}_{\mathrm{ij}}$.

$$
\begin{aligned}
& N e t_{i}=\sum_{j} w_{i j} x_{j}+s_{i} \\
& y_{i}=f\left(N e t_{i}\right)
\end{aligned}
$$

BP neural network is a typical feed forward network. It is one of the most important network of artificial neural network. A 3 layers BP neural network is shown in Fig 3, which includes input layer, hidden layer, and output layer. The neurons between adjacent layers are completely connected. But there is no connect between the neurons in the same layer [4]. The inputs are the 7 parameters, and the outputs are the axial vibration and radial vibration.

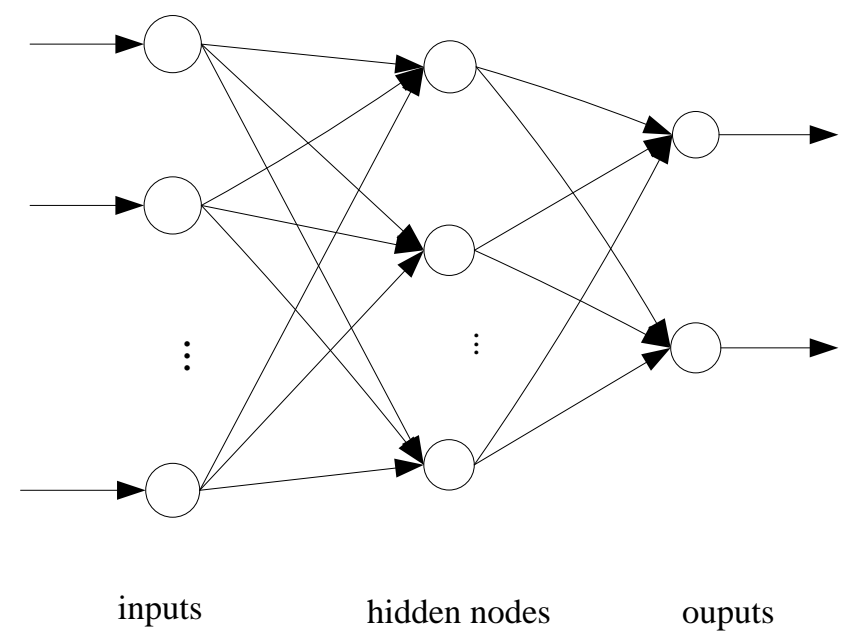

Fig.3 BP neural network

There are variety of forms of training algorithm for BP network such as the standard gradient descent algorithm, the gradient descent algorithm with momentum and the the gradient algorithm 
with variable learning rate. Every training algorithm is corresponding to a function in the matlab software.

\section{Modeling and simulation example}

Neural network construction. Neural network construction is determined by the number of input nodes, hidden nodes, hidden layers, output nodes and the transfer function. According to the analysis of the previous chapter, the number of parameters that may affect the condition of the emulsifier is 7. The number of input nodes is 7. The expected outputs of the neural network are the axial vibration and radial vibration at next time. The number of output nodes is 2 . It has been found that the neural model possesses good simulation capability when the number of hidden layers is 1 . The number of hidden nodes is set by the formula (3).

$$
s=\sqrt{m+n}+a
$$

In the formula, $\mathrm{s}$ is the number of hidden nodes, $\mathrm{m}$ is the number of input nodes, $\mathrm{n}$ is the number of output nodes. a is chose from 1 to 10 . The paper set a as 2 after several times of simulation.

Neural network training .100 groups of data are used for the neural network modeling. Every group of data contains all the parameters in the table 3-1. Considering the overfitting phenomenon, 90 groups of data are used for training, the other 10 groups are used for test.

Batch gradient descent with momentum algorithm is used for the neural network training. The corresponding function of the algorithm is Traingd function which is in the MATLAB neural net tool [6,7]. Target error is set as 0.001. Maximum iteration is set as 300. Error change is showed in the picture Fig.4.The error of the network is small than 0.001after 6 times of iteration.

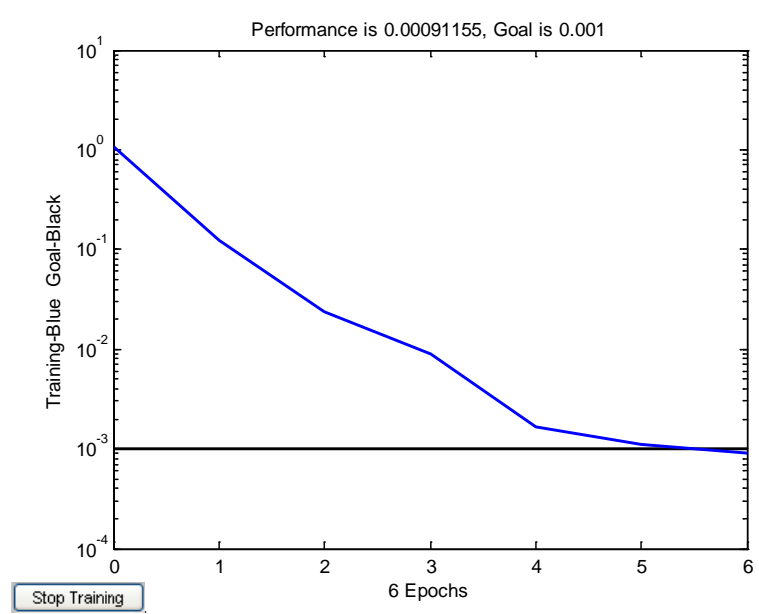

Fig.4 Error variation during the training

The test of the simulation example. The simulation is test by the 10 groups of date which are not used for training. Axial vibration of emulsifier at next time is calculated through model. In the Fig.5, little circles denote the calculation while the line denotes the actual vibration. Comparing the calculation and the actual vibration, model possesses good simulation capability. So the model can be used for emulsifier fault warning early. If the next vibration calculated by the model is abnormal, it should immediately check stands. 


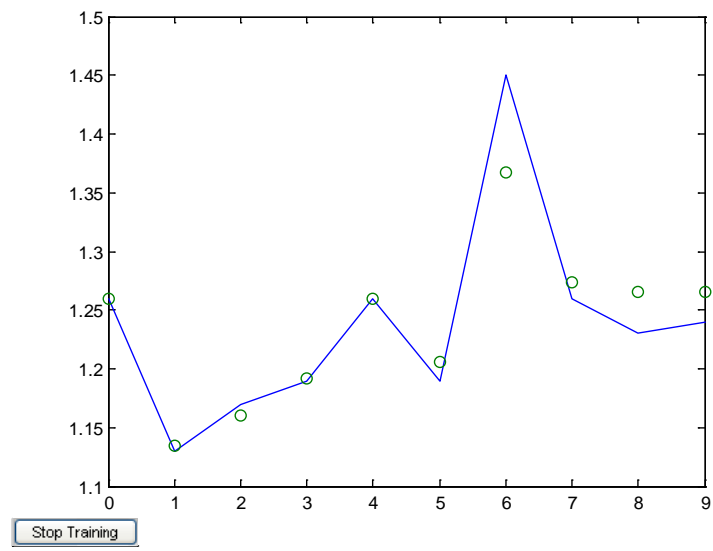

Fig.5 The comparison between calculation and actual vibration

\section{Summary}

The paper proposes a kind of emulsifier fault warning method on the BP neural network theory. The BP neural network model possesses good simulation capability through the simulation example. The vibration of emulsifier at next time can be predicted well. This method is the improvement of emulsifier threshold alarm used widely now. So the method can be promoted in the actual production.

\section{References}

[1] Xuguang Wang, Emulsion Explosives, 2nd Edition, Metallurgical Industry Press, 2008.

[2] Deyan Lv, Application of fault tree analysis for fault diagnosis in explosion of emulsifier, Mechanical \& Electrical Engineering Magazine, Vol.26,pp.24-27.

[3] Wei Wei, He Yan, Fundamentals of Intelligent Control, Tsinghua University Press, 2008,pp.30-49

[4] Zhongzhi Shi, Neural Networks, Higher Education Press,2009.

[5] Yuesheng Wang, Jun Wang, Application of Fault Tree Analysis in the Production of Emulsion Explosive, Explosive Materials, Vol.39,pp.15-17.

[6] Shifeng, Intelligence Algorithms, Beijing: Beihang University press, pp. 30-31,2011.

[7] Information on http://www.matlabsky.com. 